\title{
Bending instability characteristics of double-walled carbon nanotubes
}

\author{
Quan Wang, ${ }^{1}$ Ting $\mathrm{Hu},{ }^{2}$ Guanhua Chen, ${ }^{3}$ and Qing Jiang ${ }^{2, *}$ \\ ${ }^{1}$ Department of Mechanical, Materials and Aerospace Engineering, University of Central Florida, Orlando, \\ Florida 32816-2450, USA \\ ${ }^{2}$ Department of Mechanical Engineering, University of California, Riverside, California 92521, USA \\ ${ }^{3}$ Department of Chemistry, University of Hong Kong, Hong Kong, People's Republic of China \\ (Received 4 June 2004; revised manuscript received 1 October 2004; published 6 January 2005)
}

\begin{abstract}
The bending instability characteristics of double-walled carbon nanotubes (DWNTs) of various configurations are studied using a hybrid approach in which the deformation-induced increase of the intratube interaction energy is modeled with the bending deformation energy using the elastic theory of beams. The intertube interaction energy is calculated using the van der Waals interatomic potential. This study shows that the bending instability may take place through the formation of a single kink in the midpoint of a DWNT or two kinks, placed symmetrically about the midpoint, depending on both the tube length and diameter. The doublekink mode is more favorable for longer DWNTs with the same diameter, and there exists a threshold length for a fixed diameter, below which the single-kink mode occurs at the onset of the bending instability and above which the double-kink mode prevails. The onset characteristic of bending instability is determined by the effectiveness of the intertube interaction in transferring the load from the outer tube onto the inner tube, and the load-transfer effectiveness increases with the increasing tube length. For a fixed length/diameter ratio, the load-transfer effectiveness is found to decrease with the increasing diameter for smaller tubes while it increases for larger tubes, and, thus, the double-kink mode can prevail for both small DWNTs and large DWNTs.
\end{abstract}

DOI: 10.1103/PhysRevB.71.045403

PACS number(s): $61.46+\mathrm{w}, 46.70 . \mathrm{De}, 62.20 .-\mathrm{x}$

\section{INTRODUCTION}

Mechanical properties of carbon nanotubes (CNTs) have been a vibrant subject of research ever since the discovery of CNTs, ${ }^{1}$ which was motivated largely by many envisioned applications, such as constituents in composite materials of extremely high strength/weight ratios because of their predicted unusually high axial strength ${ }^{2-5}$ and as components of nanoscale instruments, including probes of high-resolution scanning force microscopes, ${ }^{6}$ nanobearings of superlow friction, ${ }^{7-9}$ and molecular oscillators of frequency as high as several gigahertz. ${ }^{10-14}$ There have been, for example, continuous experimental efforts ${ }^{15-18}$ to measure the axial Young's modulus and axial strength of CNTs, which have been predicted to be extremely high by various theoretical investigations, such as those cited above. We refer to a recent article of Sinnott and Andrews ${ }^{19}$ for an extensive review on this topic from the engineering perspective. Although nanoscopic systems are often perceived to be better modeled using atomic or molecular models, continuum approaches have been increasingly sought to model CNTs because atomistic simulations remain prohibitively expensive in many such investigations in which large length/diameter ratios of CNTs and/or interactions of CNTs with the external environment have to be considered, and systems involving multiwalled nanotubes (MWNTs) pose even more severe challenges to atomistic simulations. As noted by Iijima et al. ${ }^{20}$ CNTs are desired to have large length/diameter ratios for most applications in which their mechanical properties are vitally important.

Among the theoretical investigations on mechanical properties of CNTs using the continuum approach, we take particular note of the work by Yakobson and Avouris, ${ }^{21}$ in which they have shown, by comparing the predictions of the con- tinuum elastic shell model with the results from the detailed $a b$ initio and semiempirical studies of single-walled carbon nanotubes (SWNTs), that the shell model of continuum elasticity works remarkably well in modeling mechanical behaviors of CNTs, even at the limit of the linear range where instabilities occur. For modeling the mechanical instabilities of MWNTs using essentially continuum approaches, we note the work of $\mathrm{Ru}^{22,23}$ on buckling under axial compression; of Liu $e t a^{24,25}$ on rippling, a wavylike deformation mode, under bending; and of Zhang et al. ${ }^{26}$ on fracture nucleation, which are closely related to the present development of a hybrid model for kinking of double-walled nanotubes (DWNTs), an instability phenomenon taking place under severe bending. Iijima et al. ${ }^{20}$ have reported that both SWNTs and MWNTs were observed to kink under severe bending in their high-resolution electron microscopy studies. The single-kinking mode was observed for SWNTs, while both the single and double-kinking modes were observed for MWNTs. They have further studied the bending instabilities by computer simulations for both SWNTs and DWNTs, and in their simulations, the interaction between the carbon atoms within the same wall was modeled by the Tersoff Brenner many-body potential. ${ }^{27-29}$ In the case of DWNTs, the interaction between the carbon atoms in the two different walls is modeled by the van der Waals interaction potential. Their simulation results are compared with the experimental observations quite consistently for SWNTs, though not so favorably for DWNTs. We have also noted the simulations of Yakobson et al..$^{5}$ and those of Sears and Batra ${ }^{30}$ on the kinking of SWNTs and their estimate of the critical bending curvature at which kinking takes place. Furthermore, it has been proposed in literature, ${ }^{31}$ that the mechanical buckling properties of CNTs would lessen the imaging force exerted on samples in scanning probe microscopy, making CNT scan- 


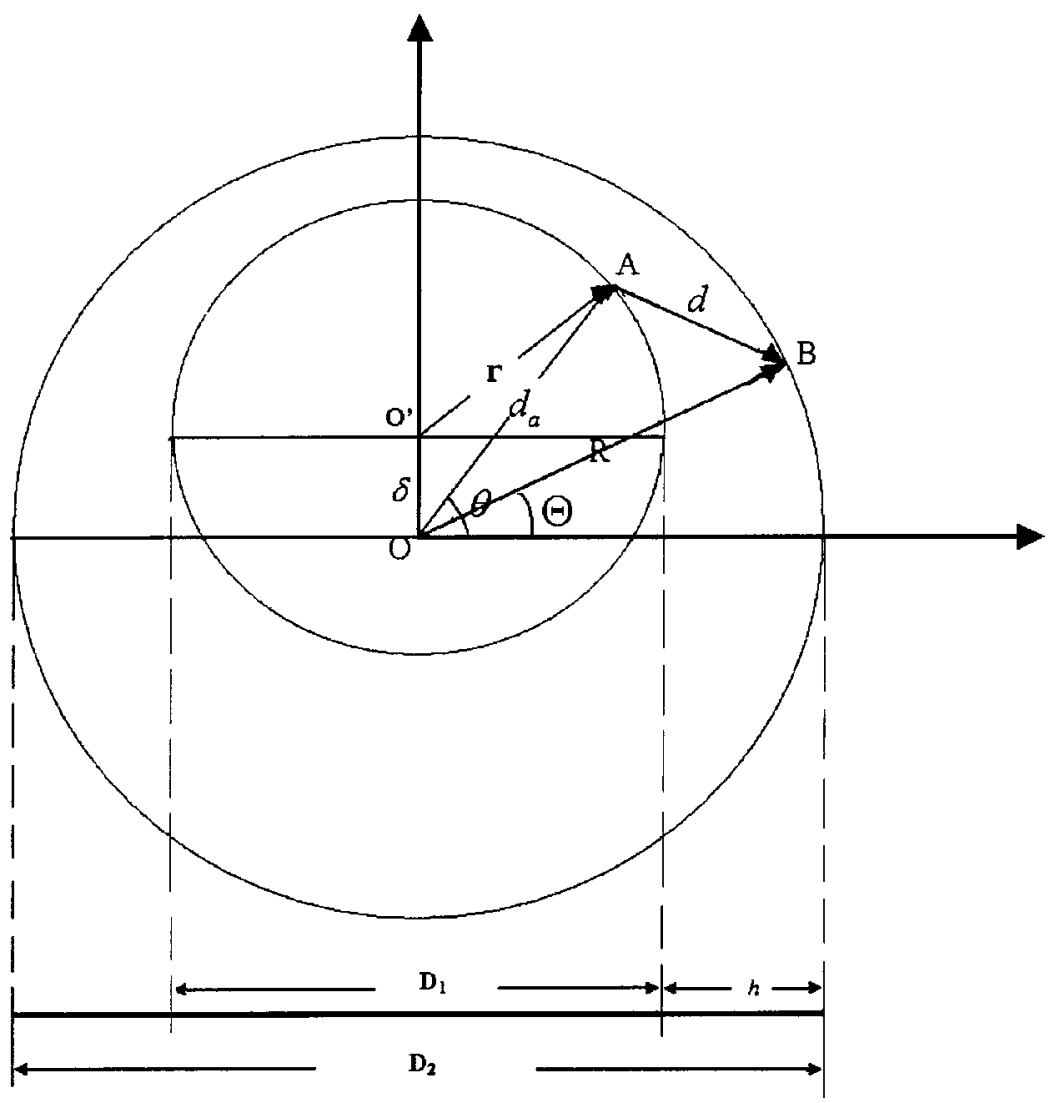

FIG. 1. Schematic illustration of a cross section of a DWNT in noncoherent bending, with $\delta$ being the eccentric distance. ning probes potentially ideal for imaging soft materials, including biological samples in liquid environments.

The simulations of Iijima et $a .^{20}$ for a DWNT of innertube diameter $D_{1}=1 \mathrm{~nm}$, outer-tube diameter $D_{2}=1.7 \mathrm{~nm}$ and of a length less than $9.26 \mathrm{~nm}$ (limited by the maximum system size of 3000 atoms) suggest that the onset of the bending instability occurs through the formation of a single kink in the middle of the DWNT and that the single kink evolves into two kinks placed symmetrically about the midpoint through a postbuckling transition upon further bending. This has brought us to question: (i) whether or not the characteristics of bending instability of a DWNT depend on its configuration parameters, the length and the diameters, in particular, or more specifically, whether or not the onset of the bending instability takes place through the formation of the two kinks, instead of a single one, for DWNTs of length and diameter different from those prescribed by Iijima et al., and (ii) if the answer is yes, then what determines whether a single kink or two kinks form in a DWNT at the onset of the bending instability. The simulations required to investigate these issues would be extremely expensive because one would need to simulate DWNTs of various configurations, some of which are very long tubes. We have therefore adopted a hybrid approach, in which the intratube interaction energy is modeled with the bending deformation energy using the elastic theory of beams and the intertube interaction energy is calculated using the van der Waals interatomic potential. Our model shows that the single-kink mode indeed appears at the onset of the bending instability for DWNTs of diameters and length/diameter ratios approximately the same as those given in the work of Iijima et al. ${ }^{20}$ while in addi- tion, it indicates that the double-kink mode prevails for longer DWNTs with the same diameter. For DWNTs of a fixed diameter, there exists a threshold length below which the single-kink mode occurs at the onset of the bending instability and above which the double-kink mode prevails. Physically, the onset characteristic of bending instability is determined by the effectiveness of the intertube interaction in transferring the load from the outer tube onto the inner tube, and the load-transfer effectiveness increases with the increasing tube length for a fixed diameter. For a fixed length/ diameter ratio, the load-transfer effectiveness is found to decrease with the increasing diameter for smaller tubes while it increases for larger tubes, and, thus, the double-kink mode can prevail for both small and large DWNTs.

\section{EFFECT OF INTERTUBE INTERACTIONS ON BENDING}

We consider a section of a DWNT of length $L$, inner and outer diameters and $D_{1}$ and $D_{2}$, and interwall spacing $h$ $=\left(D_{2}-D_{1}\right) / 2$, taken to be $0.34 \mathrm{~nm}$ (as schematically illustrated in Fig. 1) and study its bending behavior when subjected to a bending moment $M$ at each of its two ends. In this development, we characterize the bending-induced change of the interaction between atoms within the same tube by the bending-deformation energy in the continuum theory of elastic beams, and we thus model each of its two constituent tubes as a linear-elastic continuum beam of a thin-walled circular cross section. Correspondingly, the total deformation energy of the DWNT resulted from bending is given as 


$$
W=\int_{0}^{L}\left[\frac{1}{2} E I_{1}\left(\kappa_{1}\right)^{2}+\frac{1}{2} E I_{2}\left(\kappa_{2}\right)^{2}\right] d x,
$$

where $E I_{i}, i=1,2$, stand for the bending rigidities of the inner and outer tubes, with $E$ being the axial Young's modulus and $I_{i}$ the moments of inertia. $\kappa_{1}$ and $\kappa_{2}$ are the curvatures of the inner and outer tubes, respectively, within the bending plane.

We characterize the interaction between atoms of the two constituent tubes by the van der Waals interaction potential. We denote by $w_{1}(x)$ and $w_{2}(x)$, respectively, the bending deflections of the inner and outer tubes at the axial location $x$. In the case that the two tubes are bent coherently, i.e., the two constituent tubes remain concentric $\left(w_{1} \equiv w_{2}\right)$, the corresponding change in the intertube interaction energy is negligible. In the following, we estimate this change for noncoherent bending, $w_{2}>w_{1}>0$ for example, as schematically illustrated in Fig. 1, in which $\delta=w_{2}-w_{1}$ denotes the eccentric distance, and we use the following Lennard-Jones pair potential: $^{32}$

$$
\phi(d)=A\left[\frac{1}{2} \frac{\left(a d_{0}\right)^{6}}{d^{12}}-\frac{1}{d^{6}}\right],
$$

where $d$ is the interatomic distance, the van der Waals distance $d_{0}$, which is normalized by the carbon-carbon bond length $a(a=0.142 \mathrm{~nm})$, and the energy constant $A$ are taken, respectively, to be 2.362 and $24.3 \times 10^{-79} \mathrm{Jm}^{6}$, for graphite. ${ }^{32}$ We locate an atom of the inner tube by its coordinates $\left(d_{a}, \theta, z\right)$ and, correspondingly, an atom of the outer tube by $(R, \Theta, Z)$. We calculate the interaction energy $\Phi_{1}$ between a single carbon atom of the inner tube and all the atoms of the outer tube by summing Eq. (2) over all the atoms of the outer tube, and our calculations show that all the tube atoms with $|Z-z|>7 a$ together contribute less than $1 \%$ to the interaction energy $\Phi_{1}$, for the inner tube diameter $D_{1}$ ranging from $\sim 0.3$ to $100 \mathrm{~nm}$, as reported previously by Zheng et al. ${ }^{34}$ For tubes of length substantially longer than $7 a(\approx 1 \mathrm{~nm})$, we take $\Phi_{1}$ to be independent of the axial coordinate $z$, approximately. To obtain an analytical representation for $\Phi_{1}$, we use an alternative approach by replacing the discrete distribution of atoms on the outer tube with a continuous distribution of the same atom density $4 \sqrt{3} / 9 a^{2}$. We note the use of the same approach by Henrard et al. ${ }^{33}$ and Zheng and Jiang ${ }^{10}$ to calculate the intertube interaction energies, respectively, for bundles of single-walled carbon nanotubes and for a multiwalled carbon nanotube with an extruded core. This alternate approach leads to

$$
\Phi_{1}(D, \theta, \delta)=\frac{4 \sqrt{3}}{9 a^{2}} \int_{-\pi}^{\pi} \int_{-\infty}^{\infty} \phi(d) d Z R d \Theta
$$

with

$$
\begin{gathered}
d^{2}=d_{a}^{2}+\left(\frac{D+h}{2}\right)^{2}-(D+h) d_{a} \cos (\Theta-\theta)+Z^{2}, \\
\left(\frac{D-h}{2}\right)^{2}=d_{a}^{2}+\delta^{2}-2 d_{a} \delta \cos (\pi / 2-\theta) .
\end{gathered}
$$

Evaluating the integral in Eq. (3), we obtain

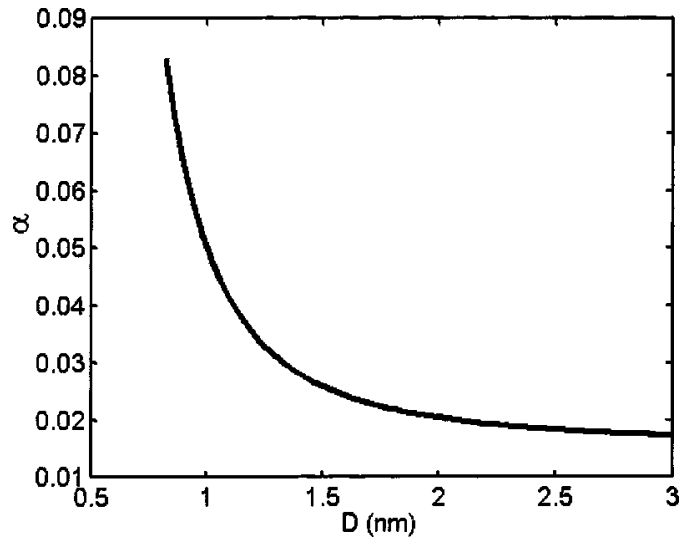

FIG. 2. Plot of the dimensionless parameter $\alpha$ vs the average diameter $D$ of DWNTs.

$$
\begin{aligned}
\Phi_{1}(D, \theta, \delta)= & \frac{7 \sqrt{3} A a^{6} d_{0}{ }^{6} \pi}{128 a^{2}}\left(\frac{D+h}{2}\right) E_{11}(D, \theta, \delta) \\
& -\frac{\sqrt{3} A \pi}{6 a^{2}}\left(\frac{D+h}{2}\right) E_{5}(D, \theta, \delta),
\end{aligned}
$$

where $D=\left(D_{1}+D_{2}\right) / 2$ is the average diameter of a DWNT, and $E_{5}$ and $E_{11}$ are two elliptical integrals defined by

$$
\begin{aligned}
E_{m}(D, \theta, \delta)= & {\left[\left(\frac{D+h}{2}\right)^{2}+d_{a}^{2}\right]^{-m / 2} } \\
& \times \int_{-\pi}^{\pi}[1+k(\theta, \delta) \cos \Theta]^{-m / 2} d \Theta
\end{aligned}
$$

with

$$
k(D, \theta, \delta)=\frac{-4(D+h) d_{a}}{(D+h)^{2}+4 d_{a}^{2}} .
$$

This leads to the intertube interaction energy per unit length of the inner tube $\Phi(\delta ; R)$ and its change $\Gamma(\delta ; D)$ corresponding to a small eccentric distance $\delta$ as follows:

$$
\begin{gathered}
\Phi(\delta ; D)=\pi A\left(\frac{D+h}{2}\right)\left(\frac{7}{96} a^{2} d_{0}{ }^{6} \int_{-\pi}^{\pi} E_{11}(D, \theta, \delta) d_{a} d \theta\right. \\
\left.\quad-\frac{2}{9 a^{4}} \int_{-\pi}^{\pi} E_{5}(D, \theta, \delta) d_{a} d \theta\right), \\
\Gamma(\delta ; D)=\Phi(\delta ; D)-\Phi(0 ; D) \approx \frac{1}{2} \alpha C D \delta^{2},
\end{gathered}
$$

where the energy constant $C=1.0 \times 10^{20} \mathrm{~J} / \mathrm{m}^{4}$ and the dimensionless parameter $\alpha=\left[\Phi^{\prime \prime}(0 ; D)\right] / C D$. We plot in Fig. 2 the parameter $\alpha$ versus the average tube diameter D, and we see that it decreases rapidly with the increasing diameter for small tubes and is nearly constant for large tubes. ${ }^{35}$ We thus obtain the following estimate for the increase of the intertube interaction energy due to bending: 


$$
U=\frac{\alpha C D}{2} \int_{0}^{L}\left[w_{1}(x)-w_{2}(x)\right]^{2} d x .
$$

In a stable configuration, the variation of the total complementary energy, defined as the sum of the intratube deformation energy $W$ and the intertube interaction energy $U$, subtracted by the total work done by all the external loads, must vanish for all the deformation variations permitted by the boundary conditions. In the present analysis, the DWNT is subjected to only a bending moment $M$ at each of its two ends of the outer tube, and, thus, the total work is given as $M\left\{\left.\left[d w_{2}(x) / d x\right]\right|_{x=L}-\left.\left[d w_{2}(x) / d x\right]\right|_{x=0}\right\}$. Approximating the bending curvatures by the second derivatives of the corresponding bending deflection functions, we obtain the following representation for the variation of the complementary energy corresponding to deflection variations $\delta w_{1}$ and $\delta w_{2}$ :

$$
\begin{aligned}
\int_{0}^{L}\{ & {\left[E I_{1} \frac{d^{4} w_{1}}{d x^{4}}+c d\left(w_{1}-w_{2}\right)\right] \delta w_{1} } \\
& \left.+\left[E I_{2} \frac{d^{4} w_{2}}{d x^{4}}+c d\left(w_{2}-w_{1}\right)\right] \delta w_{2}\right\} d x \\
& +\left.E I_{1}\left(\frac{d^{2} w_{1}}{d x^{2}}\right) \frac{d\left(\delta w_{1}\right)}{d x}\right|_{x=0, L} \\
& +\left.E I_{2}\left(\frac{d^{2} w_{2}}{d x^{2}}\right) \frac{d\left(\delta w_{2}\right)}{d x}\right|_{x=0, L}-\left.E I_{1} \frac{d^{3} w_{1}}{d x^{3}} \delta w_{1}\right|_{x=0, L} \\
& -\left.E I_{2} \frac{d^{3} w_{2}}{d x^{3}} \delta w_{2}\right|_{x=0, L}-\left.M \frac{d\left(\delta w_{2}\right)}{d x}\right|_{x=0, L}
\end{aligned}
$$

In the elastic theory of continuum beams, the second derivative of the bending deflection multiplied by the bending rigidity is equal to the bending moment, and its third derivative multiplied by the bending rigidity is identified as the shear force, i.e., the resultant transverse force acting within the cross section of the beam. Noting that the inner tube of the DWNT is subjected to neither bending moments nor transverse loads at its two ends, and that the outer tube is subjected to a bending moment $M$ at each of the two ends, we impose the following boundary conditions: for the inner tube

$$
E I_{1} \frac{d^{2} w_{1}}{d x^{2}}=0 \text { and } E I_{1} \frac{d^{3} w_{1}}{d x^{3}}=0 \text { for } x=0, L
$$

and for the outer tube

$$
E I_{2} \frac{d^{2} w_{2}}{d x^{2}}=M \text { and } E I_{2} \frac{d^{3} w_{2}}{d x^{3}}=0 \text { for } x=0, L
$$

Requiring that the variation of the complementary energy vanish for all deformation variations permitted by the boundary conditions leads to the following Euler-Lagrange equations:

$$
E I_{1} \frac{d^{4} w_{1}}{d x^{4}}=\alpha C D\left(w_{2}-w_{1}\right)
$$

$$
E I_{2} \frac{d^{4} w_{2}}{d x^{4}}=-\alpha C D\left(w_{2}-w_{1}\right) .
$$

These are the bending equations, in which the terms at the right-hand sides represent the effects of the intertube interaction on the inner and the outer model tubes in bending, which always work against noncoherent bending $\left(w_{1} \neq w_{2}\right)$. In the case $w_{2}>w_{1}>0$, for example, the interwall interaction effect on the inner tube, $\alpha C D\left(w_{2}-w_{1}\right)$, as seen in Eq. (14), works to promote the bending deflection $w_{1}$, while the effect on the outer tube, $-\alpha C D\left(w_{2}-w_{1}\right)$, reduces the bending deflection $w_{2}$. The more coherent the bending, the smaller the effect of the interwall interaction against bending, and this effect vanishes at a perfectly coherent bending configuration, in which the intertube interaction energy remains minimized.

The above bending equations remain valid until instabilities take place. In the elastic theory of continuum beams, a beam becomes buckled if further bending results in a larger increase of the deformation energy than buckling. The Euler buckling load ${ }^{21}$ for a beam structure of length $L$, moment of inertia $I$, and Young's modulus $E$, is given as $P_{c}=\pi^{2} E I / L^{2}$, and correspondingly, the in-plane critical strain for a thinwall circular beam is $\varepsilon_{c} \approx 0.5\left(\pi D_{*} / 2 L\right)^{2}$, with $D_{*}$ being the beam diameter, derived through dividing the buckling load by the beam cross-sectional area and then by the axial Young's modulus. In the classic theory of continuum beams, a beam consisting of two concentric tubes, with no intertube interactions, cannot sustain further bending when the maximum compressive strain in one of the constituent tubes reaches the corresponding critical value, while a DWNT is expected to have a higher strength against buckling because of the presence of the intertube interaction. Our hybrid model tube is therefore assumed to be capable of sustaining further bending, without inducing instabilities, after the strain in one of the two constituent tubes (the inner or the outer) becomes larger than the corresponding critical strain in one or more locations, until the strains in both the inner and outer tubes exceed the respective critical strains at the same axial location. Here we have assumed that the constitutive stress-strain relations of the two constituent tubes remain within the linear elastic limit. We note that the critical bending strain is usually taken to be twice the critical inplane strain resulted from the instability analysis of columns, considering that the strain distributes linearly along the radial direction within the tube cross section under bending while uniformly under axial compression. In Sec. III we solve the bending equations with the boundary conditions given above and determine the critical bending moment $M_{c}$ causing the onset of instabilities, when there is at least one location, denoted by $x_{k}$, where the strains of both the inner and outer tubes reach or exceed the respective critical strains, $\varepsilon_{1 c}$ $=\left(\pi D_{1} / 2 L\right)^{2}$ and $\varepsilon_{2 c}=\left(\pi D_{2} / 2 L\right)^{2}$.

There have been different choices ${ }^{25}$ for the values of the wall thickness and the axial Young's modulus defined accordingly, for the continuum shell of model nanotubes. In many continuum studies, the wall thickness and the axial Young's modulus are, respectively, taken to be the representative thickness of the graphite interplanar spacing $(\sim 0.34 \mathrm{~nm})$ and the in-plane elastic modulus of graphite 


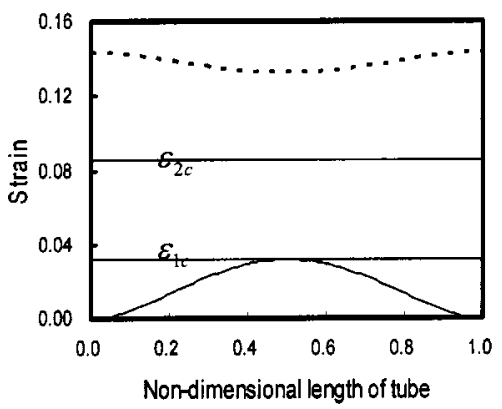

(a)

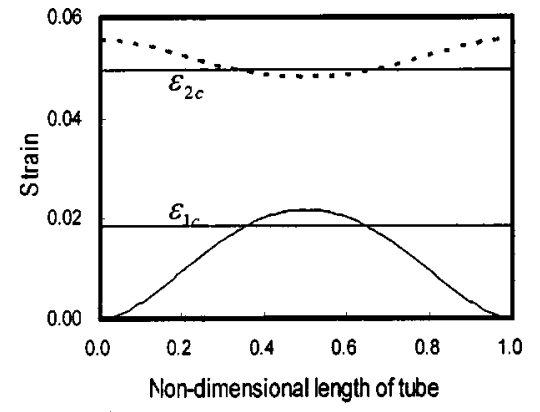

(b)
FIG. 3. Sectional maximum of the compressive strain vs the axial coordinate $x$ for both the inner and outer tubes: (a) for a DWNT of length/ diameter ratio $L / D=7$ and average diameter $D=1.35 \mathrm{~nm}$, and (b) for a DWNT of length/ diameter ratio $L / D=9.2$ and average diameter $D=1.35 \mathrm{~nm}$.
(1.06 TPa). To make the results from the continuum shell model quantitatively the same as their simulation results of atomistic models, Yakobson et al. ${ }^{21}$ have suggested that the representative thickness of the continuum shell be taken as $0.066 \mathrm{~nm}$, and, correspondingly, this leads to the Young's modulus of the continuum shell to be $5.5 \mathrm{TPa}$. We also note the suggestion of $\mathrm{Ru}^{22}$ that the flexural rigidity of each constituent be regarded as an independent material parameter, instead of a parameter derivable from the wall thickness, axial Young's modulus, and Poisson ratio. In our illustrative numerical calculations, we have followed the above suggestion of Yakobson et al. $^{21}$

\section{NUMERICAL RESULTS AND DISCUTION}

We now proceed with numerical analysis based on the following dimensionless formulation:

$$
\begin{gathered}
\frac{d^{4} \bar{w}_{1}}{d \bar{x}^{4}}=-\bar{c}_{1}\left(\bar{w}_{1}-\bar{w}_{2}\right), \\
\frac{d^{4} \bar{w}_{2}}{d \bar{x}^{4}}=-\bar{c}_{2}\left(\bar{w}_{2}-\bar{w}_{1}\right), \\
\frac{d^{2} \bar{w}_{1}}{d \bar{x}^{2}}=0 \text { and } \frac{d^{3} \bar{w}_{1}}{d \bar{x}^{3}}=0 \quad \text { for } \bar{x}=0,1, \\
\frac{d^{2} \bar{w}_{2}}{d \bar{x}^{2}}=\bar{M} \text { and } \frac{d^{3} \bar{w}_{2}}{d \bar{x}^{3}}=0 \quad \text { for } \bar{x}=0,1,
\end{gathered}
$$

where $\bar{x}=x / L, \bar{w}_{1}=w_{1} / D, \bar{w}_{2}=w_{2} / D, \bar{c}_{1}=\alpha C D L^{4} /\left(E I_{1}\right), \bar{c}_{2}$ $=\alpha C D L^{4} /\left(E I_{2}\right)$, and $\bar{M}=M L^{2} /\left(E I_{2} D\right)$. We note that one has to fix at least two points of the beam in the numerical calculations to avoid rigid rotations and translations. We have therefore required that the deflection of the outer tube vanish at the both ends and have verified in all the calculations that the share forces at the both ends vanish identically, as required by the second boundary condition stated in Eq. (19).

Iijima et $a{ }^{20}$ have reported that the onset of bending instabilities in their simulations of a DWNT took place as a single kink formed at the midpoint of the DWNT, for which the diameters of the inner wall and the outer wall are given to be $D_{1}=1.0 \mathrm{~nm}$ and $D_{2}=1.7 \mathrm{~nm}$, respectively. Although they did not specify the length of their nonhelical DWNT, we have estimated that the length of their model SWNT should be shorter than $9.26 \mathrm{~nm}$, considering their statement that their simulations are limited to no more than 3000 atoms. We, thus, first consider a DWNT of an average diameter $D$ $=1.35 \mathrm{~nm}\left[\approx 0.5\left(D_{1}+D_{2}\right)\right]$ and a length/diameter ratio $L / D$ $=7$, and then we calculate the maximum compressive strains at each cross section of axial coordinate $x$, for both the inner and outer tubes, and for each prescribed bending moment. We have found that the maximum compressive strain at every cross section of the outer tube exceeds the critical strain $\varepsilon_{2 c}$, before the maximum compressive strain of the inner tube reaches its critical value $\varepsilon_{1 c}$ at any of its cross sections. Examining the sectional maximum of the compressive strain versus the axial coordinate $x$ for both the inner and outer tubes reveals that the sectional maximum for the outer tube decreases monotonically from its maxima at the two ends to its minima at the midpoint, while the sectional maximum for the inner tube increases monotonically from zero at its two ends to the maxima occurring at the midpoint, both being symmetric about the midpoint as expected. On further increasing the bending moment to $M_{c r}=676 \mathrm{eV}$, the global maximum compressive strain of the inner tube occurring at its midpoint exceeds its critical value, as shown in Fig. 3(a), leading to instability, and, thus, a single kink forms at the midpoint of the modeling beam for the DWNT. Correspondingly, the critical bending angle $\theta_{c r}=M_{c r} L /(E I)$ has an approximate value $71^{\circ}$.

To examine the effect of the tube diameter on the bending instability, we gradually increase the length/diameter ratio from $L / D=7$, while keeping the average diameter the same (i.e., $D=1.35 \mathrm{~nm}$ ) and find that, until $L / D>8.2$, the onset characteristic of the bending instability remains the same (i.e., occurrence of a single kink at the midpoint of the beam), although the critical bending moment deceases with the increasing length/diameter ratio. The reduction of the critical bending moment has two causes: (i) the critical compressive strains decrease quadratically with the increasing length/diameter ratio and (ii) the inner tube shares increasingly more bending load with the increasing length because the external bending moments are applied on the outer tube and the loads are transferred onto the inner tube over the length through the intertube interaction. After the length/ diameter ratio exceeds a threshold value $L / D=8.2$, a transition in the bending instability mode occurs and the onset of the bending instability takes place through the formation of two kinks, placed symmetrically about the midpoint of the 
beam, instead of a single kink at the midpoint. In the further simulation, the profiles of the sectional maximum of the compressive strain versus the axial coordinate remain the same for both the inner and outer tubes, but the global maximum of the compressive strain of the inner tube, however, exceeds its critical value at the bending moment $M$ $=224 \mathrm{eV}$ before its outer tube counterpart reaches the corresponding critical value. As the bending moment increases from 224 to $237 \mathrm{eV}$, the global maxima of the outer tube occurring at the two ends increase correspondingly to reach the critical value, meanwhile the inner tube has developed a middle portion, within which the sectional maximum of the compressive strain at every cross section has exceeded its critical value. At this point, the model beam is capable of sustaining further bending because there is not a single cross section at which the sectional maximum compressive strains of both the inner and outer tubes have reached their respective critical values. On further increase of the bending moment to $M_{c r}=267 \mathrm{eV}$, we find two cross sections, located symmetrically with respect to the midpoint of our model beam, in which the sectional maximum compressive strains of both the inner and outer tubes have reached their respective critical values, as shown in Fig. 3(b), leading to the formation of two kinks. The corresponding critical bending angle $\theta_{c r}$ is $37^{\circ}$. Our further calculations show that the distance separating the two kinks increases with the increasing length/diameter ratio, when the average diameter is kept constant.

We turn now to reveal the effect of the diameter of the DWNT at a fixed-length/diameter ratio on the onset of the bending instability. We note, from the above simulations, that whether the single- or double-kink mode occurs at the onset of the bending instability, this corresponds to whether the maximum compressive strain at the midpoint of the outer tube reaches the outer tube critical strain before that of the inner tube reaches the corresponding critical strain or vice versus. Noting the fact that the critical strains for both the inner and outer tubes vary insignificantly with the average diameter $D$ at a fixed-length/diameter ratio and for the average diameter $D$ much larger than the interwall spacing $h$ (Ref. 36), we see that the bending instability mode is determined by the effectiveness of the intertube interaction in transferring the load from the outer tube to the inner tube. The more effective the intertube interaction in the load transfer, the larger the increase in the maximum compressive strain is at the midpoint of the inner tube in responding to

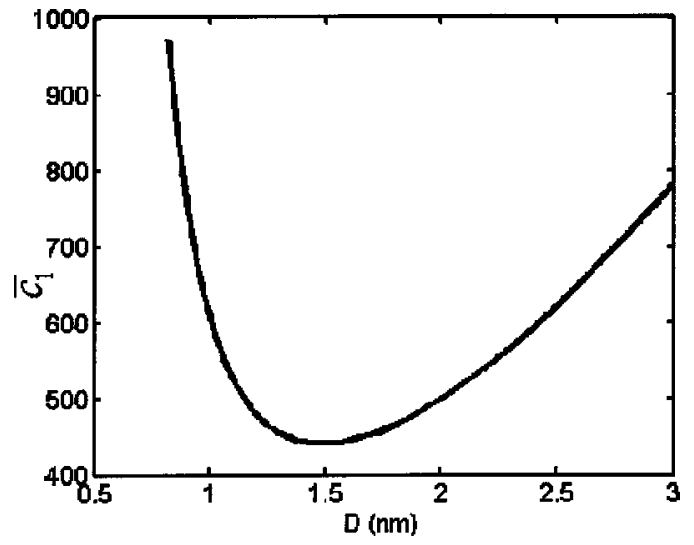

FIG. 4. Plot of the dimensionless parameter $\bar{c}_{1}$ vs the diameter for DWNTs of length/diameter ratio $L / D=8.4$.

further bending. We note from the dimensionless formulation [Eqs. (16)-(19)], that the dimensionless interaction parameter $\bar{c}_{1}$ is the primary indicator for the effectiveness of the intertube interaction in transferring the load from the outer tube to the inner tube and find that this parameter decreases with the diameter for small DWNTs and increases with the diameter for larger DWNTs, and the turning point varies with the length/diameter ratio. We plot in Fig. 4 the dimensionless interaction parameter $\bar{c}_{1}$ versus the diameter for DWNTs of the length/diameter ratio $L / D=8.40$, slightly larger than the threshold length/diameter ratio, 8.20, above which the double-kink mode occurs at the onset of bending instability and below which the single-kink mode takes place. As seen from Fig. 4, the dimensionless interaction parameter $\bar{c}_{1}$ decreases with the increasing diameter from 1.35 to $1.50 \mathrm{~nm}$, and this decrease is shown to be significant enough to cause the transition of the onset of bending instability from the double-kink mode to the single-kink mode, as illustrated in Fig. 5(a), which shows that the maximum compressive strain at the midpoint of the inner tube reaches its critical value after that of the outer tube has exceeded the corresponding critical value for a DWNT of diameter $D=1.5 \mathrm{~nm}$ and length/diameter ratio $L / D=8.40$, leading to a single kink at the midpoint. The dimensionless interaction parameter $\bar{c}_{1}$ is seen in Fig. 4 to increase with the further increasing diameter from $1.5 \mathrm{~nm}$, and this increase is expected to lead to the transition reversal, i.e., from the single-kink mode back to the double-kink mode when it becomes significantly large, as it is shown in Fig. 5(b) that the maximum compressive strain
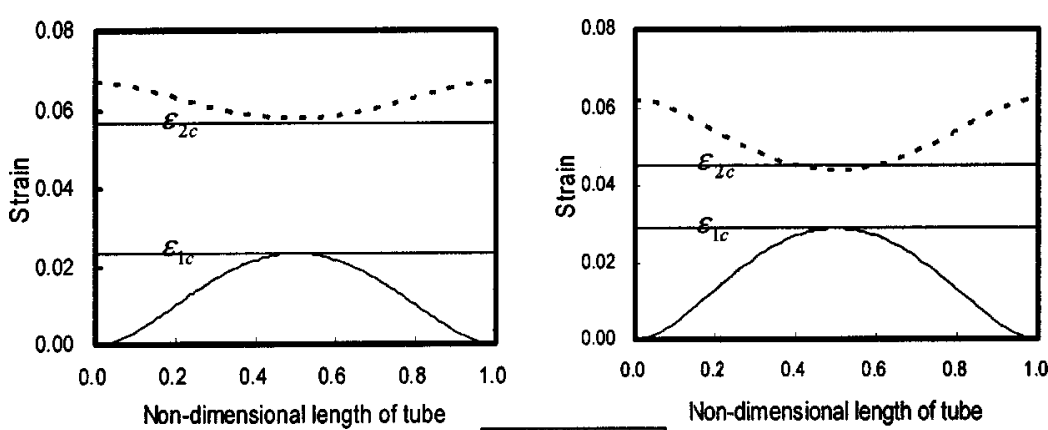

(a)

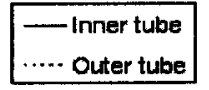

Non-dimensional length of tube

(b)
FIG. 5. Sectional maximum of the compressive strain vs the axial coordinate $x$ for both the inner and outer tubes for a DWNT of length/ diameter ratio $L / D=8.4$ (a) at $D=1.5 \mathrm{~nm}$ the maximum compressive strain at the midpoint of the inner tube reaches its critical value after that of the outer tube has exceeded the corresponding critical value, leading to a single kink at the midpoint; (b) at $D=3 \mathrm{~nm}$ the maximum compressive strain at the midpoint of the inner tube reaches its critical value before that of the outer tube reaches the corresponding critical value, leading to two kinks upon further bending. 
at the midpoint of the inner tube reaches its critical value before that of the outer tube has exceeded the corresponding critical value for a DWNT of diameter $D=3.0 \mathrm{~nm}$ and length/diameter ratio $L / D=8.40$, and that this would lead to the double-kink mode when the onset of bending instability occurs upon further bending.

In conclusion, we present a hybrid model for a DWNT subjected to bending in which the bending-induced change of the interaction between atoms within the same wall is described as the bending deformation energy in the continuum theory of elastic beams, while the corresponding change in the interaction between atoms of two constituent walls is characterized by the van der Waals interaction potential. This simple model indicates that the bending instability may take place through the formation of a single kink at the midpoint of the beam or two kinks placed symmetrically about the midpoint of the beam, depending on both the tube length and diameter. These two distinct modes of bending instability have both been observed by Iijima et al.,${ }^{20}$ and for a DWNT of average diameter $1.35 \mathrm{~nm}$ and length/ diameter ratio less than 7, their atomistic simulations suggest that the bending instability will take place by forming a single kink at the midpoint of the DWNT, although the double-kink mode becomes energetically favorable over the single-kink mode upon further bending. The present analysis confirms that the single-kink mode indeed appears at the onset of the bending instability for the DWNT of the given diameter and length/diameter ratio, while in addition, it indicates that the double-kink mode prevails for longer DWNTs of the same diameter. For DWNTs of a fixed diameter, there exists a threshold length below which the single-kink mode occurs at the onset of the bending instability and above which the double-kink mode prevails. Physically, the onset characteristic of bending instability is determined by the effectiveness of the intertube interaction in transferring the load from the outer tube onto the inner tube, and the loadtransfer effectiveness increases with the increasing tube length for a fixed diameter. For a fixed-length/diameter ratio, the load-transfer effectiveness is found to decrease with the increasing diameter for smaller tubes while increase for larger tubes, and, thus, the double-kink mode can prevail for both small and large DWNTs. We have attempted to compare our predictions with HRTEM images of bent CNTs in literature, but this comparison has not led to meaningful conclusions because experiment data for kinking of double-walled CNTs, including lengths and diameters, do not appear to be available. We have found some excellent HRTEM images of the bent CNTs of more than two walls and are planning to generate our analysis to CNTs of more than two walls. We further note that the postinstability characteristics, such as the takeover of the double-kink mode to the single-kink mode upon further bending indicated by the simulations of Iijima et al.,$^{20}$ cannot be analyzed with this simple model because of the limitation of the linear analysis employed.

\section{ACKNOWLEDGMENT}

The support of the U.S. National Science Foundation through research Grant No. CMS-0140568 is gratefully acknowledged.
*Corresponding author. Email address: qjiang@engr.ucr.edu

${ }^{1}$ S. Iijima, Nature (London) 354, 56 (1991).

${ }^{2}$ D. H. Roberton, D. W. Brenner, and J. W. Mintmire, Phys. Rev. B 45, 12592 (1992).

${ }^{3}$ P. Calvert, Nature (London) 357, 365 (1992).

${ }^{4}$ M. M. J. Treacy, T. W. Ebbesen, and J. M. Gibson, Nature (London) 381, 678 (1996).

${ }^{5}$ B. I. Yakobson, C. J. Brabec, and J. Bernholc, Phys. Rev. Lett. 76, 2511 (1996).

${ }^{6}$ S. Akita, H. Nishijima, Y. Nakayama, F. Tokumasu, and K. Takeyasu, J. Phys. D 32, 1044 (1999).

${ }^{7}$ R. E. Tuzun, D. W. Noid, and B. G. Sumpter, Nanotechnology 6, 64 (1995).

${ }^{8}$ J. Cumings and A. Zettl, Science 289, 602 (2000).

${ }^{9}$ B. Bourlon, D. C. Glattli, C. Miko, L. Forro, and A. Bachtold, Nano Lett. 4, 709 (2004).

${ }^{10}$ Q. S. Zheng and Q. Jiang, Phys. Rev. Lett. 88, 045503 (2002).

${ }^{11}$ Y. Zhao, C. C. Ma, G. H. Chen, and Q. Jiang, Phys. Rev. Lett. 91, 175504 (2003).

${ }^{12}$ S. B. Legoas, V. R. Coluci, S. F. Braga, P. Z. Coura, S. O. Dantas, and D. S. Galvao, Phys. Rev. Lett. 90, 055504 (2003).

${ }^{13}$ J. L. Rivera, C. McCabe, and P. T. Cummings, Nano Lett. 3, 1001 (2003)

${ }^{14}$ W. L. Guo and Y. F. Guo, Phys. Rev. Lett. 91, 125501 (2003).

${ }^{15}$ G. Gustafsson, Y. Cao, and G. M. Treacy, Nature (London) 357,
477 (1992).

${ }^{16}$ E. W. Wong, P. E. Sheehan, and C. M. Lieber, Science 277, 1971 (1997).

${ }^{17}$ P. Poncharal, Z. L. Wang, D. Ugarte, and W. A. de Heer, Science 283, 1513 (1999).

${ }^{18}$ M. F. Yu, O. Lourie, M. J. Dyer, K. Moloni, T. F. Kelly, and R. S. Ruoff, Science 287, 637 (2000).

${ }^{19}$ S. B. Sinnott and R. Andrews, Crit. Rev. Solid State Mater. Sci. 26, 145 (2001).

${ }^{20}$ S. Iijima, C. Brabec, A. Maiti, and J. Bernholc, J. Chem. Phys. 104, 2089 (1996).

${ }^{21}$ B. I. Yakobson and P. Avouris, Top. Appl. Phys. 80, 287 (2001).

${ }^{22}$ C. Q. Ru, Phys. Rev. B 62, 9973 (2000).

${ }^{23}$ C. Q. Ru, J. Appl. Phys. 89, 3426 (2001).

${ }^{24}$ J. Z. Liu, Q. S. Zheng, and Q. Jiang, J. Appl. Phys. 86, 4843 (2001).

${ }^{25}$ J. Z. Liu, Q. S. Zheng, and Q. Jiang, Phys. Rev. B 67, 075414 (2003).

${ }^{26}$ P. Zhang, Y. Huang, H. Gao, and K. C. Hwang, ASME J. Appl. Mech. 69, 454 (2002).

${ }^{27}$ J. Tersoff, Phys. Rev. Lett. 56, 632 (1986).

${ }^{28}$ J. Tersoff, Phys. Rev. Lett. 61, 2879 (1988).

${ }^{29}$ D. W. Brenner, Phys. Rev. B 42, 9458 (1990).

${ }^{30}$ A. Sears and R. C. Batra, Phys. Rev. B 69, 235406 (2004).

${ }^{31}$ R. M. Stevens, C. V. Nguyen, and M. Meyyappan, IEEE Trans. 
Nanobiosci. 3, 56 (2004).

${ }^{32}$ B. T. Kelly, Physics of Graphite (Applied Science Publishers, London, 1981), p. 153.

${ }^{33}$ L. Henrard, E. Hernandez, P. Bernier, and A. Rubio, Phys. Rev. B 60, R8521 (1999).

${ }^{34}$ Q. S. Zheng, J. Z. Liu, and Q. Jiang, Phys. Rev. B 65, 245409 (2002).

${ }^{35}$ We note that $\mathrm{Ru}$ has approximated $\Gamma(\delta ; R)$ by $c D \delta^{2} / 2$, with $c$ $\approx 1.0 \times 10^{19} \mathrm{erg} / \mathrm{cm}^{4}$, based on his estimate using the interaction between two flat graphite sheets: C. Q. Ru, J. Appl. Phys. 89, 3426 (2001); Int. J. Solids Struct. 36, 869 (1999).

${ }^{36}$ The in-plane critical strain is defined as $\varepsilon_{c}=P_{c} /$ EA where $P_{c}$ $=\pi^{2} E I / L^{2}$ is the Euler buckling load for a beam of the Young's modulus $E$, cross-sectional area $A$, inertia moment $I$, and length
$L$. For a thin-wall circular beam, $I \approx \pi D_{*}^{3} t / 8$ ( $t$ is the thickness), $A \approx \pi D_{*} t$, with $D_{*}$ being the diameter of the thin wall, and we thus have the in-plane critical strain $\varepsilon_{c} \approx 0.5\left(\pi D_{*} / 2 L\right)^{2}$, and the corresponding bending critical strain $\varepsilon_{c} \approx\left(\pi D_{*} / 2 L\right)^{2}$. For the inner and outer walls of a double-walled CNT, with $D_{1}=D-h$ and $D_{2}=D+h$, respectively, this leads to the following expressions for the bending critical strains: $\varepsilon_{1 c}=\left(\pi D_{1} / 2 L\right)^{2}$ and $\varepsilon_{2 c}$ $=\left(\pi D_{2} / 2 L\right)^{2}$. Alternatively, $\varepsilon_{1 c}=(\pi D / 2 L)^{2}(1-h / D)^{2}$ and $\varepsilon_{2 c}$ $=(\pi D / 2 L)^{2}(1+h / D)^{2}$ with $D$ being the average diameter of the double-walled NT, and it is seen that they both vary insignificantly with the average diameter $D$ at a fixed length/diameter ratio $(D / L)$ and for the average diameter $D$ much larger than the interwall spacing $h$. 